Series A

I. MATHEMATICA

402

\title{
BOUNDARY VALUES AND INJECTIVENESS OF THE SOLUTIONS OF BELTRAMI EQUATIONS
}

BY

OLLI MARTIO

H E L S I N K I 1967

S U O M A L A I N E T TEDEAKATEM A

doi:10.5186/aasfm.1967.402 
Communicated 10 February 1967 by Olli Lehto and K. I. Virtanen 


\section{Preface}

I am deeply indebted to Professor Olli Lehto and Professor I. S. Louhivaara for suggesting this subject and for their kind interest and valuable advice.

I also wish to thank the Finnish Scientific Society for financial support.

Helsinki, February 1967.

Olli Martio 


\section{Introduction}

In this paper we study the following problem: Given Beltrami differential equations in an arbitrary Jordan region $G$ of the complex plane $\mathbf{C}$, how to choose the boundary values for the real part of the solution $w=u+i v$ such that the mapping $w: G \rightarrow \mathbf{C}$ is injective. This problem was first treated by Morrey [7] in a special case. We give a simple general condition for the boundary values of $u$ which guarantees the injectiveness of $w$.

For the sake of completeness, we solve in part 1 the first boundary value problem associated with the second-order Beltrami equation $L(u)=0$. This is done by Perron's method without the global reduction of $L(u)=0$ to the canonical form $\Delta u=0$.

\section{Elliptic boundary value problem for the Beltrami equation}

In this part the boundary value problem associated with the secondorder Beltrami differential equation (cf. [4] p. 66)

$$
a u_{x_{1} x_{1}}+2 b u_{x_{1} x_{2}}+c u_{x_{2} x_{2}}+\left(a_{x_{1}}+b_{x_{2}}\right) u_{x_{1}}+\left(b_{x_{1}}+c_{x_{2}}\right) u_{x_{2}}=0,
$$

where $a c-b^{2}=1$, is solved under general conditions on the coefficients and boundary data. For this we use Perron's method (cf. [9]), well-known in the treatment of the classical Dirichlet problem.

\subsection{Notations and preliminaries}

By $\mathbf{R}$ we denote the set of real numbers. Points in two dimensional plane $\mathbf{R}^{2}$ are denoted by $x, y, \ldots$ or $\left(x_{1}, x_{2}\right),\left(y_{1}, y_{2}\right), \ldots$ In the plane the usual norm topology is used.

If $F$ is a subset of $\mathbf{R}^{2}$, then $\bar{F}$ is the closure and $\partial F$ the boundary of $F$. The set $B(x, R)$ is the open ball in $\mathbf{R}^{2}$ with center at $x$ and radius $R$. Sometimes one or both arguments in $B$ are dropped.

The restriction of the mapping $f: F \rightarrow \mathbf{R}$ (or $\mathbf{R}^{2}$ ) to $F^{\prime} \subset F$ is denoted by $f \mid F^{\prime}$. The set $C^{0}(F)$ is the set of ail continuous mappings on $F$. We say that $f$ belongs to $C^{n}(F), n=1,2, \ldots$, if there exists an 
open set $U$ such that $U \supset F$ and the partial derivatives of order $n$ of $f$ belong to $C^{0}(U)$. All $C^{n}(F), n=1,2, \ldots$, are vector spaces over $\mathbf{R}$.

If $f \in C^{0}(F)$ and

$$
\sup _{\substack{x, y \in F \\ x \neq y}} \frac{|f(x)-f(y)|}{|x-y|^{\mu}}=\|f\|_{H_{\mu}(F)} \quad(0<\mu<1)
$$

is finite, then $f$ is said to beiong to the class $C_{\mu}^{0}(F)$, the set of all Höldercontinuous functions with exponent $\mu$ on $F$. The set of functions for which the $n$th partial derivatives are Hölder-continuous with exponent $\mu$ is denoted by $C_{\mu}^{n}(F)$. Here $\|f\|_{H_{\mu}(F)}$ is a seminorm on $C_{\mu}^{0}(F)$. If $F$ is compact, e.g. $C^{0}(F), C_{\mu}^{0}(F)$, and $C_{\mu}^{1}(F)$ are Banach-spaces with norms

$$
\begin{aligned}
& \|f\|_{C^{0}(F)}=\sup _{x \in F}|f(x)| \\
& \|f\|_{C_{\mu}^{0}(F)}=\|f\|_{C^{0}(F)}+\|f\|_{H_{\mu}(F)}, \\
& \|f\|_{C_{\mu}^{1}(F)}=\|f\|_{C^{0}(F)}+\sum_{i=1}^{2}\left\|f_{x_{i}}\right\|_{C_{\mu}^{0}(F)} .
\end{aligned}
$$

We shall need some sets of singular functions. Denote by $F / F$ the set

$$
F \times F-\{(x, y) \mid x=y\} \text {. }
$$

We say that $Z: F / F \rightarrow \mathbf{R}$ belongs to $S^{p}(F), p>0$, if

$$
\sup _{(x, y) \in F / F}|Z(x, y)||x-y|^{p}=\|Z\|_{S p(F)}
$$

is finite. It is clear that $S^{p}(F) \subset S^{q}(F)$, if $p<q$ and $F$ is bounded.

Remark 1.1.1. If $Z \in S^{p}(F), p>1, F$ is compact, and $f \in C^{1}(F)$, then

$$
Z^{\prime}(x, y)=(f(x)-f(y)) Z(x, y)
$$

belongs to $S^{p-1}(F)$ and

$$
\left\|Z^{\prime}\right\|_{S{ }^{p-1}(F)} \leqq\|f\|_{C^{1}(F)}\|Z\|_{S p_{(F)}} \cdot
$$

In this connection we cite the lemma [4] p. 185:

Lemma 1.1.1. Let $D$ be a compact, convex set in $\mathbf{R}^{2}, f \in C^{0}(D)$, and $Z \in S^{1+\alpha}(D), 0<\alpha<1$. If $Z$ is a continuous function on $D / D$ and

$$
|Z(x, y)-Z(z, y)| \leqq\left[\frac{C_{1}}{\mid x-y !^{2}}+\frac{C_{2}}{|z-y|^{2}}\right]|x-z|^{1-\alpha}
$$

$\left(C_{1}\right.$ and $C_{2}$ constants) for all $x, z$, and $y$ in $D$ which satisfy

$$
|x-y|>2|x-z|>0,
$$


then

$$
g(x)=\iint_{D} Z(x, t) f(t) d t_{1} d t_{2}=\int_{D} Z(x, t) f(t) d t
$$

belongs to $C_{\gamma}^{0}(D)$, if $0<\gamma<1-\alpha$.

Note that the integral in (1.1.2) is an absolutely convergent Riemann integral.

Remark 1.1.2. (Cf. [4] p. 186.) The inequality (1.1.1) is satisfied, if the derivatives $Z(x, y)_{x_{i}}, i=1,2$, are continuous and belong to $S^{2+\alpha}(D)$.

\subsection{Construction of a locally homeomorphic solution for the Bers system}

Our aim is to construct a locally homeomorphic solution for the pair of equations

$$
\begin{aligned}
& v_{x_{2}}=a u_{x_{1}}+b u_{x_{2}}, \\
& v_{x_{1}}=-\left(b u_{x_{1}}+c u_{x_{2}}\right)
\end{aligned}
$$

under the conditions

$$
\begin{gathered}
a, b, c \in C_{\mu}^{1}(\bar{B}), \\
\Delta(x)=a(x) c(x)-b(x)^{2}>0, a(x)>0, x \in \bar{B},
\end{gathered}
$$

where $B=B(z, R)$. The equations are then called a Bers system. If in addition $\Delta=1$, then (1.2.1) are called Beltrami equations. The construction of a locally homeomorphic solution of Beltrami equations has been carried out, e.g. Ahlfors [1], Bojarski [10], and Lehto-Virtanen [5].

By eliminating $v$ from (1.2.1) we get for $u$ the equation

$$
L(u)=\left(a u_{x_{1}}+b u_{x_{2}}\right)_{x_{1}}+\left(b u_{x_{1}}+c u_{x_{2}}\right)_{x_{2}}=0 .
$$

With the solution of (1.2.4) we understand a function $u \in C^{2}(B)$ which satisfies (1.2.4) in $B$.

To find a locally homeomorphic sclution for (1.2.1) we at first construct a family of solutions of (1.2.4). We start with the so called parametrix

$$
\begin{aligned}
P(x, y)=-\frac{1}{4 \pi \sqrt{ }} & =\frac{1}{\Delta(y)} \log \left\{\frac { 1 } { \Delta ( y ) } \left[c(y)\left(x_{1}-y_{1}\right)^{2}\right.\right. \\
& \left.\left.\quad-2 b(y)\left(x_{1}-y_{1}\right)\left(x_{2}-y_{2}\right)+a(y)\left(x_{2}-y_{2}\right)^{2}\right]\right\},
\end{aligned}
$$

which is a continuous function on $\bar{B} / \bar{B}$. If in the following some partial derivatives of the parametrix appear, then they are always taken with respect to the first argument of $P$. They are continuous functions defined on $\bar{B} / \bar{B}$. 
We need some estimates which control the growth of $P(x, y)$ when $|x-y|$ tends to zero; for this reason we define positive constants $\alpha, \beta$, and $E_{0}$ :

$$
\begin{aligned}
& \alpha=\max \left(\|a\|_{c_{\mu}^{1}(\bar{B})},\|b\|_{C_{\mu}^{1}(\bar{B})},\|c\|_{C_{\mu}^{1}(\bar{B})}\right) \\
& \beta=\inf \sqrt{\Delta(x)}
\end{aligned}
$$

and $E_{0}$ is the constant of ellipticity, i.e. the greatest lower bound of all $\gamma$ such that

$$
c(y) x_{1}^{2} \pm 2 b(y) x_{1} x_{2}+a(y) x_{2}^{2} \geqq \gamma\left(x_{1}^{2}+x_{2}^{2}\right), x, y \in \bar{B} .
$$

The constant $E_{0}$ is positive on the basis of (1.2.3).

Lemma 1.2.1. (Cf. e.g. [4] p. 181.) The parametrix $P$ has the following properties:

a) $P \in S^{p}(\bar{B})$ for any $p>0$,

b) $P_{x_{i}} \in S^{1}(\bar{B})$ and $\left\|P_{x_{i}}\right\|_{S^{1}(\bar{B})} \leqq \alpha\left(\beta E_{0}\right)^{-1}, \quad i=1,2$,

c) $P_{x_{i} x_{j}} \in S^{2}(\bar{B})$ and $\left\|P_{x_{i} x_{j}}\right\|_{S^{2}(\bar{B})} \leqq \alpha^{2} \beta^{-1} E_{0}^{-2}, i, j=1,2$,

d) $L\left(P, \in S^{1}(\bar{B})\right.$ and $\|L(P)\|_{S^{1}(\bar{B})} \leqq 4 \alpha^{2} \beta^{-1} E_{0}^{-2}\left(\alpha / E_{0}+1\right)$.

Proof: Assertions a), b) and c) are obvious.

To prove d) write

$$
L(P)=a P_{x_{1} x_{1}}+2 b P_{x_{1} x_{2}}+c P_{x_{2} x_{2}}+A,
$$

where $A$ stands for terms which contain the first partial derivatives of $P$. These terms clearly belong to $S^{1}(\bar{B})$. By direct computation it can be shown that

$$
a(y) P(x, y)_{x_{1} x_{1}}+2 b(y) P(x, y)_{x_{1} x_{2}}+c(y) P(x, y)_{x_{2} x_{2}}=0
$$

in $\bar{B} / \bar{B}$, hence

$$
\begin{aligned}
L(P(x, y))= & (a(x)-a(y)) P(x, y)_{x_{1} x_{1}}+2(b(x)-b(y)) P(x, y)_{x_{1} x_{2}} \\
& +(c(x)-c(y)) P(x, y)_{x_{2} x_{2}}+A(x, y) .
\end{aligned}
$$

By c) $P_{x_{i} x_{j}}$ belongs to $S^{2}(\bar{B})$, therefore by Remark 1.1.1 we obtain, after elementary calculation, $\mathrm{d}$ ).

Next we study mappings $T_{0}$ and $T$ defined as

$$
\begin{gathered}
\left(T_{0} u\right)(x)=\int_{\bar{B}} P(x, t) u(t) d t, \\
T v u=v+T_{0} u .
\end{gathered}
$$


By Lemma 1.2.1 a) the integral in (1.2.5) is an absolutely convergent Riemann integral, if $u \in C^{0}(\bar{B})$. According to b) the same is true if we replace $P$ by $P_{x_{i}}(i=1,2)$, and so

$$
\left(T_{0} u\right)(x)_{x_{i}}=\int_{\bar{B}} P(x, t)_{x_{i}} u(t) d t
$$

We have proved

Lemma 1.2.2. $T_{0}$ is a bounded linear map from $C^{0}(\bar{B})$ into $C^{1}(\bar{B})$, and $T$ maps $C_{\gamma}^{2}(\bar{B}) \times C^{0}(\bar{B})$ into $C^{1}(\bar{B})$.

Now we introduce a special Cauchy principal value (cf. [6] p. 24). If $Z \in S^{2}(\bar{B})$, and

$$
E(x, \varrho)=\left\{y \mid c(x) y_{1}^{2}-2 b(x) y_{1} y_{2}+a(x) y_{2}^{2} \leqq \varrho^{2} \Delta(x)\right\}
$$

denotes »the fundamental ellipse», then we define

$$
\lim _{\varrho \rightarrow 0} \int_{\bar{B}-E(x, \varrho)} Z(x, t) d t=\int_{\bar{B}}^{*} Z(x, t) d t,
$$

if the limit exists. The following lemma can be found in [6] p. 24:

Lemma 1.2.3. $T_{0}$ maps $C_{\gamma}^{0}(\bar{B}), 0<\gamma<1$, into $C^{2}(B)$, and the following formulas are valid:

$$
\left(T_{0} u\right)(x)_{x_{i} x_{j}}=A_{i j}(x)+\int_{\bar{B}}^{*} P(x, t)_{x_{i} x_{j}} u(t) d t,
$$

$i, j=1,2$, where

$$
\begin{gathered}
A_{11}=-\frac{c}{2 \Delta} u, \quad A_{22}=-\frac{a}{2 \Delta} u, \\
A_{12}=A_{21}=\frac{b}{2 \Delta} u .
\end{gathered}
$$

The two previous lemmas imply immediately:

Corollary 1.2.1. $T$ maps $C_{\gamma}^{2}(\bar{B}) \times C_{\gamma}^{0}(\bar{B})$ into $C^{2}(B)$.

From Lemma 1.2.3 we get the generalized Poisson equation as corollary.

Corollary 1.2.2. If $u$ belongs to $C_{\gamma}^{0}(\bar{B})$, then

$$
L\left(\left(T_{0} u\right)(x)\right)=-u(x)+\int_{\bar{B}} L(P(x, t)) u(t) d t .
$$

Note that the integral in (1.2.8) is taken in the ordinary sense because of Lemma 1.2.1 d). 
Corollary 1.2.3. Let $f \in C_{\gamma}^{2}(\bar{B})$ be given. If $u \in C_{\gamma}^{0}(\bar{B})$, then

$$
u(x)=L(f(x))+\int_{\bar{B}} L(P(x, t)) u(t) d t
$$

if and only if $L(T f u)=0$ in $B$.

Proof: This is a direct application of (1.2.8) to the last term in the right side of

$$
L(T f u)=L(f)+L\left(T_{0} u\right) .
$$

Theorem 1.2.1. For given $f$ in $C_{\gamma}^{2}(\bar{B}), 0<\gamma<\mu$, there exists a uniquely determined $u$ in $C_{\gamma}^{0}(\bar{B})$ with the property

$$
L((T f u)(x))=0, x \in B(z, R),
$$

if $R$ is sufficiently small.

Proof: According to Corollary 1.2.3 we must solve the integral equation (1.2.9), which is a singular equation of Fredholm's type.

Define $D: C^{0}(\bar{B}) \rightarrow C^{0}(\bar{B})$ as (cf. Lemma 1.2.1)

$$
(D w)(x)=L(f(x))+\int_{\bar{B}} L(P(x, t)) w(t) d t .
$$

The mapping $D$ is a contraction from the Banach-space $C^{0}(\bar{B})$ into itself for $R$ small enough. We see this with the help of Lemma 1.2.1 as follows:

$$
\begin{aligned}
\| D w & -D w^{\prime}\left\|_{C^{0}(\bar{B})} \leqq\right\| w-w^{\prime} \|_{C^{0}(\bar{B})} \int_{\overline{\bar{B}}}|L(P(x, t))| d t \\
& \leqq\left\|w-w^{\prime}\right\|_{C^{0}(\bar{B})}\|L(P)\|_{S^{1}(\bar{B})} \int_{\overline{\bar{B}}} \frac{1}{|x-t|} d t \\
& \leqq 4 \pi R\|L(P)\|_{S^{1}(\bar{B})}\left\|w-w^{\prime}\right\|_{c^{0}(\bar{B})},
\end{aligned}
$$

and

$$
4 \pi R\|L(P)\|_{S^{1}(\bar{B})}
$$

can be chosen smaller than 1 by choosing $R$ small enough. Hence there exists a uniquely determined $u$ in $C^{0}(\bar{B})$ with the property $u=D u$.

To prove that $u$ belongs to $C_{\gamma}^{0}(\bar{B})$ we use Lemma 1.1.1. Clearly $L(f)$ belongs to $C_{\gamma}^{0}(\bar{B})$, hence we must consider

$$
\left(T^{\prime} u\right)(x)=\int_{\bar{B}} L(P(x, t)) u(t) d t .
$$


The function $L(P) \in S^{1}(\bar{B})$ consists of two different parts, $L(P)=B+A$, where (cf. Lemma 1.2.1)

$$
\begin{gathered}
B(x, y)=(a(x)-a(y)) P(x, y)_{x_{1} x_{1}}+2(b(x)-b(y)) P(x, y)_{x_{1} x_{2}} \\
+(c(x)-c(y)) P(x, y)_{x_{2} x_{2}}, \\
A(x, y)=\left(a(x)_{x_{1}}+b(x)_{x_{2}}\right) P(x, y)_{x_{1}}+\left(b(x)_{x_{1}}+c(x)_{x_{2}}\right) P(x, y)_{x_{2}} .
\end{gathered}
$$

According to Remark 1.1.1 we can use Remark 1.1.2 and it follows that

$$
\left(T_{B}^{\prime} u\right)(x)=\int_{\bar{B}} B(x, t) u(t) d t
$$

maps $C^{0}(\bar{B})$ into $C_{\gamma}^{0}(\bar{B})$. To prove that $T_{A}^{\prime}$ does the same, estimate

$$
\begin{aligned}
& \left|A(x, t)-A\left(x^{\prime}, t\right)\right| \\
& \leqq\left|a(x)_{x_{1}}+b(x)_{x_{2}}-a\left(x^{\prime}\right)_{x_{1}}-b\left(x^{\prime}\right)_{x_{2}}\right|\left|P(x, t)_{x_{1}}\right| \\
& \quad+\left|a\left(x^{\prime}\right)_{x_{2}}+b\left(x^{\prime}\right)_{x_{2}}\right|\left|P(x, t)_{x_{1}}-P\left(x^{\prime}, t\right)_{x_{1}}\right|+\ldots,
\end{aligned}
$$

where dots stand for two similar terms. The first term in the right hand side of (1.2.10) is less than

$$
2 \alpha\left\|P_{x_{1}}\right\|_{S^{1}(\bar{B})} \frac{\left|x-x^{\prime}\right|^{\mu}}{|x-t|},
$$

and to the second term we can apply Remark 1.1.2, hence (1.1.1) holds with $\alpha=1-\mu$. By Lemma 1.1.1

$$
T^{\prime} u=\left(T_{B}^{\prime}+T_{A}^{\prime}\right) u, u \in C^{0}(\bar{B}),
$$

belongs to $C_{\gamma}^{0}(\bar{B})$, if $\gamma<\mu$. But

$$
u=L(f)+T^{\prime} u,
$$

hence $u \in C_{\gamma}^{0}(\bar{B})$, which proves the theorem.

Theorem 1.2.1 shows that there corresponds to every $f$ in $C_{\gamma}^{2}(\bar{B})$ a unique $u=H f$ in $C_{\gamma}^{0}(\bar{B})$. It is clear that the mapping $H$ is linear.

Remark 1.2.1. The function $u$ in the previous theorem can be found by successive approximations:

$$
\begin{gathered}
u_{0}=L(f), \\
u_{n+1}(x)=L(f(x))+\int_{\bar{B}} L(P(x, t)) u_{n}(t) d t, n=0,1, \ldots,
\end{gathered}
$$

and for the norm of $u=\lim u_{n}$ we have the usual estimate

$$
\|u\|_{C^{0}(\bar{B})}=\|H f\|_{C^{0}(\bar{B})} \leqq \frac{C_{1}}{1-C_{2} R},
$$


where

$$
C_{1}=\|L(f)\|_{C^{0}(\bar{B})}, \quad C_{2}=4 \pi\|L(P)\|_{S^{1}(\bar{B})} .
$$

Next we prove the existence of a locally homeomorphic solution for a Bers system; in fact we show that there exists a whole family of them. By a locally homeomorphic solution we mean a mapping

$$
w=(u, v): B(z, R) \rightarrow \mathbf{R}^{2}, \quad u, v \in C^{2}(B),
$$

which satisfies (1.2.1) in $B$ and is injective.

Theorem 1.2.2. If $f$ belongs to $C_{\gamma}^{2}(\overline{B(z, R)})$ and

$$
k=f(z)_{x_{1}}^{2}+f(z)_{x_{2}}^{2}>0,
$$

then there exists a locally homeomorphic solution $w=(u, v)$ of (1.2.1) for all sufficiently small values of $R$, with the property $u=T f H f$.

Proof: By Theorem 1.2.1 the function $u=T f H f \in C^{2}(B)$ is a solution of (1.2.4). Hence the function $v$ can be integrated from (1.2.1), because this system is exact. Moreover, $v$ belongs to $C^{2}(B)$.

For the Jacobian of the mapping $w=(u, v)$ the following estimate holds at $z$ :

$$
\begin{aligned}
|J(w(z))| & =\left|a(z) u(z)_{x_{1}}^{2}+2 b(z) u(z)_{x_{1}} u(z)_{x_{2}}+c(z) u(z)_{x_{2}}^{2}\right| \\
& \geqq E_{0}\left(u(z)_{x_{1}}^{2}+u(z)_{x_{2}}^{2}\right) .
\end{aligned}
$$

At $z$ we have

$$
u(z)=f(z)+\int_{\bar{B}} P(z, t)(H f)(t) d t
$$

hence

$$
\left|u(z)_{x_{i}}\right| \geqq\left|f(z)_{x_{i}}\right|-\int_{\bar{B}}\left|P(z, t)_{x_{i}}\right||(H f)(t)| d t, \quad i=1,2 .
$$

Using Lemma 1.2.1 and Remark 1.2.1 we get

$$
\int_{\bar{B}}\left|P(z, t)_{x_{i}}\right||(H f)(t)| d t \leqq\left\|P_{x_{i}}\right\|_{S^{1}(\bar{B})} \frac{C_{1}}{1-C_{2} R} \int_{\bar{B}} \frac{d t}{|z-t|} \leqq \text { constant } R,
$$

therefore

$$
\left[u(z)_{x_{1}}\right]^{2}+\left[u(z)_{x_{2}}\right]^{2} \geqq k-\text { constant } R \geqq \frac{k}{2},
$$

if $R$ is small enough. Hence the Jacobian of $w$ is positive at $z$, and so the mapping $w$ is injective for all sufficiently small values of $R$. This proves the theorem. 


\subsection{Solution of the boundary value problem by Perron's method}

By the first boundary value problem associated with the Beltrami equation (A) we mean the following: To find a function $u$ defined in the closure of a region $G \subset \mathbf{R}^{2}$ such that $u \in C^{2}(G) \cap C^{0}(\bar{G}), u$ satisfies (A) in $G$, and $u$ is equal to a given function $g$ on the boundary $\partial G$ of $G$.

From the coefficients in (A) it is assumed that (1.2.2), (1.2.3), and $\Delta=1$ hold, when $B$ is replaced by some bounded region $G$ in $\mathbf{R}^{2}$.

In the following we present a generalization of Perron's method, which can be used to solve Dirichlet's problem in the plane. Our method is based on the general principle that if we know the solution of the boundary value problem in the small, we get a solution in the large (cf. [1]).

We begin with some definitions.

The mapping $w=(u, v): F \rightarrow \mathbf{R}^{2}, F \subset G$, is called pseudoanalytic in the region $F$, if $u$ and $v$ belong to $C^{2}(F)$ and the pair $(u, v)$ is a solution of the Beltrami equations (1.2.1) in $F$. The function $u$ is called pseudoharmonic and $v$ is called the pseudoconjugate of $u$. Both $u$ and $v$ are solutions of second-order, self-adjoint partial differential equations, especially is $L(u)=0$ in $F$.

In the previous section we have shown that every point $z \in \bar{G}$ has a neighbourhood $B(z, R)$, where a pseudoanalytic mapping exists which is also a homeomorphism for all sufficiently small values of $R$. For a fixed $z$ we denote this mapping by $W$ and its inverse mapping by $W^{-1}$.

In the following lemmas we prove some well-known results (cf. [5] and [10]), which show that pseudoharmonic functions have analogous properties with harmonic functions.

Lemma 1.3.1. Every pseudoanalytic mapping $w$ can be locally expressed in the form

$$
w=\Phi \circ W,
$$

where $\Phi$ is an analytic function on the image of $W$. Conversely if $\Phi$ is analytic, then (1.3.1) is pseudoanalytic in the domain of $W$.

Proof: By direct computation it can be shown that $\Phi=w \circ W^{-1}$ is a solution of the Cauchy-Riemann differential equations.

Corollary 1.3.1. If $v$ is harmonic on the image of $W$, then $v \circ W$ is pseudoharmonic, and if $u$ is pseudoharmonic, then $u \circ W^{-1}$ is harmonic.

Lemma 1.3.2. If $u$ is in the region $F$ pseudoharmonic, then $u$ obeys the maximum principle on $F$, that is: if $u$ takes its maximum value in $F$, then it is constant.

Proof: Let $u$ attain its maximum value at the point $z$ of $G$ and let $B(z, R) \subset G$ be so small that $W$ exists on $B$. The maximum principle for harmonic functions implies that the function $u \circ W^{-1}$, which is harmonic by Corollary 1.3.1, is constant on the region $W(B)$, hence $u$ is 
constant. Because $F$ is connected the above result can be generalized to the whole of $F$.

Since $u$ and $-u$ are simultaneously pseudoharmonic, $u$ cannot take its minimum in $F$.

Lemma 1.3.3. (Generalized Harnack's principle.) Let $u_{i}, i=1,2, \ldots$, be a monotonic sequence $\left(u_{i} \leqq u_{j}, i<j\right)$ of pseudoharmonic functions on the region $F$. Then on every compact subset $K$ of $F$, either

$$
\lim _{i \rightarrow \infty} u_{i}=+\infty,
$$

or the sequence $u_{i}$ converges uniformly towards a pesudoharmonic function $u$.

Proof: Let $z \in F$ and take $R$ so small that $W$ exists on $\overline{B(z, R)}$. By Corollary 1.3.1 the sequence $u_{i} \circ W^{-1}$ is a monotonic sequence of harmonic functions, hence by the principle of Harnack for harmonic functions, either

$$
\lim _{i \rightarrow \infty} u_{i} \circ W^{-1}=+\infty,
$$

or the sequence $u_{i} \circ W^{-1}$ converges uniformly to a harmonic function $h$ on the set $\overline{W(B)}$. On $\bar{B}$, the lemma is proved, because $h \circ W$ is pseudoharmonic there. By covering $K$ with a finite number of circles $B\left(z_{j}, R_{j}\right)$, the above result can be extended to the whole of $K$.

Perron used subharmonic functions to solve the classical Dirichlet problem. This method can be easily generalized to our situation.

Definition 1.3.1. A continuous function $w$ defined on the region $F$ is called subpseudoharmonic, if the function $w-u$, where $u$ is pseudoharmonic in an open set $U \subset F$, obeys the maximum principle in $U$.

Remark 1.3.1. The above definition has a local character: If we define the function $w$ to be subpseudoharmonic at the point $x$ if $w$ is subpseudoharmonic in some open neighbourhood $U$ of $x$, then $w$ is subpseudoharmonic in $F$ if and only if it is subpseudoharmonic at each point $x$ in $F$.

Every pseudoharmonic function is subpseudoharmonic.

Lemma 1.3.4. If $u_{1}, u_{2}, \ldots, u_{n}$ are subpseudoharmonic functions and $i a$ positive real number, then the functions $\lambda u_{1}, u_{1}+u_{2}$, and $\max \left(u_{1}, u_{2}, \ldots, u_{n}\right)$ are all subpseudoharmonic.

Proof: Obviously the functions $\lambda_{2} u_{1}$ and $u_{1}+u_{2}$ are subpseudoharmonic.

The function $w=\max \left(u_{1}, u_{2}\right)$ is subpseudoharmonic. Firstly it is continuous. Secondly if the difference between a pseudoharmonic function $u$ and the function $w$ takes its maximum at the point $z$, then one of the functions, for instance $u_{1}$, has the property

$$
u_{1}-u=\text { constant. }
$$


But

$$
w(x)-u(x) \leqq w(z)-u(z)=u_{1}(z)-u(z)=u_{1}(x)-u(x) \leqq w(x)-u(x)
$$

in a neighbourhood of the point $z$, and hence by the maximum principle

$$
w-u=\text { constant. }
$$

By induction it follows that $\max \left(u_{1}, u_{2}, \ldots, u_{n}\right)$ is subpseudoharmonic.

For the boundary value problem the regularity of the boundary of the region $G$ plays an essential role.

Definition 1.3.2. The boundary point $z$ of the region $G$ is called regular, if there exists a subpseudoharmonic function $v$, called barrier, such that

$$
\lim _{x \rightarrow z, x \in G} v(x)==0 \text {. }
$$

and $v(x) \leqq k(\varepsilon)<0$, when $x \in G-B(z, \varepsilon)$.

Other boundary points are called irregular. Some sufficient conditions for the regularity of a boundary point are given later.

Theorem 1.3.1. Let $G$ be a bounded region in $\mathbf{R}^{2}, D$ the set of all regular boundary points of $\partial G$, and let $g$ belong to $C^{0}(\partial G)$. Under these assumptions here exists a pseudoharmonic function $u$ such that

$$
\lim _{x \rightarrow z, x \in G} u(x)=g(z),
$$

when $z \in D$.

Proof: Let $S_{g}(G)$ be the set of all subpseudoharmonic functions $w$ such that

$$
\lim _{x \rightarrow y} \sup u(x) \leqq g(y), \quad x \in G, y \in \partial G .
$$

The set $S_{g}(G)$ is not empty, because all sufficiently small constants belong to it. We claim that

$$
u_{g}=\sup _{u \in S_{g}(G)} u
$$

is the pseudoharmonic function mentioned in the theorem.

a) At first we prove that $u_{g}$ is pseudoharmonic.

Let $y$ be a fixed point in $G$ and $B(y, \varepsilon) \subset G$ so small that the local homeomorphism $W$ exists in $\overline{B(y, \varepsilon)}$. We denote by $P(f, B(y, \varepsilon)) x$ the Poisson integral

$$
P(f, B(y, \varepsilon)) x=(2 \pi)^{-1} \int_{0}^{2 \pi} \frac{\left(\varepsilon^{2}-|x-y|^{2}\right) f\left(y_{1}+\varepsilon \cos \Theta, y_{2}+\varepsilon \sin \Theta\right)}{\varepsilon^{2}-2|x-y| \varepsilon \cos (\Theta-\varphi)+|x-y|^{2}} d \Theta,
$$


where $x_{1}-y_{1}=|x-y| \cos \varphi, x_{2}-y_{2}=|x-y| \sin \varphi$, and $f$ is a function belonging to $C^{0}(\partial B)$. It is well-known that $P(f, B) x$ is a harmonic function in $B$.

Let $\delta$ be so small that $B(W(y), \delta)$ is contained in $W(B(y, \varepsilon))$. The function $w^{*}$ is called an improvement relative to $B(W(y), \delta)$ of the subpseudoharmonic function $w \in S_{g}(G)$ in $G$, if

$$
\begin{aligned}
& w^{*}(x)=P\left(w \circ W^{-1}, B(W(y), \delta)\right) W(x), \quad x \in W^{-1}(B(W(y), \delta)), \\
& w^{*}(x)=w(x), \quad x \in G-W^{-1}(B(W(y), \delta)) .
\end{aligned}
$$

The function $w^{*}$ belongs to $S_{g}(G)$ : Because

$$
\lim _{x \rightarrow z} P(f, B) x=f(z), \quad x \in B, z \in \partial B,
$$

we obtain

$$
\lim _{x \rightarrow z} w^{*}(x)=P\left(w \circ W^{-1}, B(W(y), \delta)\right) W(z)=w \circ W^{-1} \circ W(z)=w(z),
$$

when $x$ belongs to $W^{-1}\left(B(W(y), \delta)\right.$ and $z$ to $\partial W^{-1}(B(W(y), \delta))$; hence $w$ is continuous. In addition we remark that $w^{*} \mid W^{-1}(B(W(y), \delta))$ is pseudoharmonic and so $w^{*}-u$ certainly obeys the maximum principle in $W^{-1}(B(W(y), \delta))$ and in $G-\overline{W^{-1}(B(W(y), \delta))}$, too. To prove that the maximum principle for $w^{*}-u$ is satisfied on $\partial W^{-1}(B(W(y), \delta))$, suppose that $w^{*}-u$ has a maximum at $z \in \partial W^{-1}(B(W(y), \delta))$. Then also $w-u$ has a maximum at $z$, hence $w-u$ is constant. It follows easily from the Poisson integral representation in (1.3.1) that $w \leqq w^{*}$. Therefore

$$
w(x)-u(x) \leqq w^{*}(x)-u(x) \leqq w^{*}(z)-u(z)=w(x)-u(x)
$$

in some neighbourhood of $z$, and so $w^{*}-u$ is constant.

Let $w_{i}, i=1,2, \ldots$, be a sequence of subpseudoharmonic functions in the set $S_{g}(G)$ such that

$$
\lim _{i \rightarrow \infty} w_{i}(y)=u_{g}(y), \quad y \in G .
$$

By Lemma 1.3.4 the sequence can be taken to be increasing, for it can be replaced by the sequence

$$
\max \left(w_{1}, w_{2}, \ldots, w_{i}\right), \quad i=1,2, \ldots
$$

We replace every $w_{i}$ by its improvement $w_{i}^{*}$ relative to $B(W(y), \delta)$. The functions $w_{i}$ are pseudoharmonic in $W^{-1}(B(W(y), \delta))$ and form an increasing sequence. By the generalized Harnack's principle the limit

$$
u_{*}=\lim _{i \rightarrow \infty} w_{i}^{*}
$$


exists on every compact subregion of $W^{-1}(B(W(y), \delta))$ and defines there a pseudoharmonic function $u_{*}$. Note that $\lim w_{i}^{*}$ cannot be $+\infty$, for every $w_{i}^{*}$ is smaller than or equal to $\sup g(x)$, which is finite. In addition $u_{*}$ is equal to $u_{g}$ at $y$, for

$$
w_{i}(y) \leqq w_{i}^{*}(y) \leqq u_{g}(y)
$$

Next we prove that $u_{*}=u_{g}$ in some neighbourhood of the point $y$. After this the proof of the part a) is complete, because we have shown that $u_{g}$ is pseudoharmonic in a neighbourhood of each point $y$ in $G$, hence $u_{g}$ is pseudoharmonic in $G$.

Take a point $x$ so close to $y$ that the set $B(W(x), \lambda)$ contains $W(y)$ for some $\lambda>0$, and

$$
B(W(x), \lambda) \subset B(W(y), \delta) .
$$

Let $w_{i}^{\prime}$ be an increasing sequence of subpseudoharmonic functions such that

$$
\lim _{i \rightarrow \infty} w_{i}^{\prime}(x)=u(x)
$$

We put

$$
f_{i}=\max \left(w_{i}^{\prime}, w_{i}^{*}\right)
$$

and denote by $f_{i}^{*}$ the improvement of $f_{i}$ relative to $B(W(x), \lambda)$. By the generalized Harnack's principle the limit

$$
f_{*}=\lim f_{i}^{*}
$$

is pseudoharmonic on $W^{-1}(B(W(x), \lambda))$. By the definition of the function $f_{i}$

$$
u_{*} \leqq f_{*}
$$

in $W^{-1}(B(W(x), \lambda))$. But by the definition of $u_{g}$

$$
f_{*}(y) \leqq u_{g}(y)=u_{*}(y)
$$

hence the pseudoharmonic function

$$
\left(f_{*}-u_{*}\right) \mid W^{-1}(B(W(x), \lambda))
$$

takes its minimum zero in $W^{-1}(B(W(x), \lambda))$. It follows that

$$
f_{*}-u_{*}=0
$$

in $W^{-1}(B(W(x), \lambda))$. Hence we have

$$
u_{*}(x)=u_{g}(x),
$$


and so the above equality holds in some neighbourhood of $y$. The first part of the theorem is proved.

b) The second step is to prove that for $y \in D$

$$
\lim _{x \rightarrow y, x \in G} u_{g}(x)=g(y) \text {. }
$$

At first we take such a neighbourhood $B(y, R)$ of the point $y$ that

$$
|g(y)-g(x)|<\varepsilon,
$$

when $x \in \partial G \cap B(y, R)$. Because $y$ is a regular boundary point there exists a subpseudoharmonic function $v$ with the property

$$
\sup _{x \in G-B(y, \varepsilon)} v(x) \leqq k(\varepsilon)<0 .
$$

Because $G$ is a bounded region a constant $\lambda$ can be found such that the subpseudoharmonic function

satisfies

$$
u(x)=\lambda v(x)+g(y)-\varepsilon
$$

$$
\lim _{x \rightarrow z} \sup w(x) \leqq g(z)
$$

for all $z \in \partial G$, hence $w \in S_{g}(G)$. Because

we get

$$
u_{g}=\sup _{u \in S_{g}(G)} u
$$

$$
\lim _{x \rightarrow y} \inf u_{g}(x) \geqq \lim _{x \rightarrow y} w(x)=g(y)-\varepsilon .
$$

Next we show that the corresponding inequality holds for the limes superior.

From the construction of the function $w$ it follows that for all $u \in S_{g}(G)$

$$
\lim _{x \rightarrow z} \sup (u(x)+\lambda v(x)-g(y)-\varepsilon) \leqq 0,
$$

when $z \in \partial G$ and $\lambda$ is large enough. By Lemma 1.3.4 the function $u+\lambda v$ is subpseudoharmonic, hence

$$
u(x) \leqq-\lambda v(x)+g(y)+\varepsilon
$$

by the maximum principle at every point $x \in G$. This estimate is true for all $u \in S_{g}(G)$, therefore

(1.3.4) $\quad \lim _{x \rightarrow y} \sup u_{g}(x) \leqq \lim _{x \rightarrow y}(-\lambda v(x)+g(y)+\varepsilon)=g(y)+\varepsilon$. 
The estimates (1.3.3) and (1.3.4) hold for all $\varepsilon>0$ at every regular boundary point $y$ of $\partial G$, hence

$$
\lim _{x \rightarrow y} u_{g}(x)=g(y), \quad x \in G, y \in D,
$$

and the theorem is proved.

We now go back to consider the properties of regular boundary points. In fact it is clear that if the boundary point of the region is regular for the classical Dirichlet problem, it is regular for the boundary value problem of pseudoharmonic functions. This is due to the fact that all barriers constructed for the first problem can be generalized with the help of the local homeomorphism $W$ to the latter problem. We also remark that the existence of the barrier is a local property of the boundary curve.

By means of Riemann's mapping theorem the following theorem is easily established.

Theorem 1.3.2. If $\partial G$ is a Jordan curve, then there exists a barrier at every point $y \in \partial G$.


We denote by $K$ the connected component of $B(y, R) \cap G$ which contains $y$. Let $J$ be a Jordan arc which is defined as the connected component of $\partial K \cap \partial G$ containing $y$.

Let $\Phi$ be the conformal transformation which maps $W(K)$ onto $B(0,1)$. It is well known that $\Phi$ is continuous up to the boundary $\partial K$, for this is a Jordan curve. Now we construct the function $v$ defined as

$$
\begin{aligned}
& v \mid K=P(f, B(0,1)) \Phi \circ W, \\
& v \mid(\bar{G}-K)=-1,
\end{aligned}
$$

where $f$ is continuous on $\partial B(0,1)$,

$$
f \mid \Phi \circ W(\partial K-J)=-1,
$$

$-1 \leqq f \leqq 0$, and $f$ is not zero exept at the point $y$. Clearly $v(x)<0$, $x \in \bar{G}, x \neq y, v(y)=0$, and $v$ is continuous on $\bar{G}$. Moreover $v$ is subpseudoharmonic on $G$, for on $K$ it is pseudoharmonic by Corollary 1.3.1 and also on $G-\bar{K}$, because it is constant there. Suppose now that

$$
w(x)=v(x)-u(x),
$$

$u$ pseudoharmonic, takes its maximum $-1-u(z)$ on $\partial K \cap G$ at the point z. Denote by $U$ the neighbourhood of the point $z$ such that $w(x) \leqq w(z)$ in $U$. Then

$$
w(x)=-1-u(x) \leqq-1-u(z),
$$


when $x$ belongs to $U \cap(G-K)$. In $U \cap K$ we have

$$
-1-u(z)=v(z)-u(z) \geqq v(x)-u(x) \geqq-1-u(x),
$$

for $v \geqq-1$ in $U \cap K$. By (1.3.5) and (1.3.6)

$$
u(x) \geqq u(z),
$$

when $x \in U$, hence by the minimum principle for pseudoharmonic functions $u=$ constant, and so $w=$ constant. The theorem is proved.

Corollary 1.3.2. On a Jordan region $G$ there exists one and only one pseudoharmonic function $u$ such that $u \in C^{0}(G)$ and $u$ is equal to a given func. tion $g \in C^{0}(\partial G)$ on $\partial G$.

Proof: The existence of $u$ follows from Theorem 1.3.1 and 1.3.2. The uniqueness is a direct consequence of the maximum principle.

Remark 1.3.2. The boundary value problem can be generalized to certain cases in which $g$ is not continuous on $\partial G$ (cf. [9]). Also Theorem 1.3.2 remains true, if the boundary $\partial G$ is not free.

We also remark that Corollary 1.3.2 is not true, if $G$ is unbounded although its boundary consists only of regular points.

\section{Homeomorphic solutions for Beltrami equations}

\subsection{The problem and preliminaries}

In this part we study the following problem: Given an arbitrary Jordan region $G$ in $\mathbf{R}^{2}$, how to choose such boundary values for $u$ in $(1.2 .1)$ that the mapping $w=(u, v)$ is a homeomorphism from $G$ onto $w(G)$. This problem was treated by Morrey [7] p. 140 in a special case.

For the sake of simplicity we use complex nocation $x=x_{1}+i x_{2}$ $\left(i^{2}=-1\right)$ for the points in $\mathbf{R}^{2}$. The Beltrami equations (1.2.1) take the form

$$
c w_{x_{2}}-(-b+i) w_{x_{1}}=0 \text {, }
$$

where $w=u+i v$.

We denote by $B(G)$ the set of all mappings $w=u+i v$ such that $u \in C^{2}(G) \cap C^{0}(\bar{G}), \quad v \in C^{2}(G)$, and $w$ satisfies (2.1.1) in $G$.

As a summary from the previous part we have

Theorem 2.1.1. If $G$ is a Jordan region, the assumptions (1.2.2), (1.2.3), and $A=1$ hold in $\bar{G}$, and $g \in C^{0}(\partial G)$, then there exists $w=u+i v$ in $B(G)$ with the property $u \mid \partial G=g$. The mapping $w$ is uniquely determined up to an imaginary constant.

Proof: By Corollary 1.23 we can find a function $u$ such that $u$ belongs to $C^{2}(G) \cap C^{0}(\bar{G}), \quad u \mid \partial G=g$, and $L(u)=0$. Then $v \in C^{2}(G)$ can be integrated from (1.2.1), because this system is exact. 


\subsection{The case of continuous boundary values}

The first two lemmas and corollary presented in this section are wellknown (cf. for instance [10]).

Lemma 2.2.1. Suppose that $w$ is a non-constant pseudoanalytic function in the region $G$. Then the zeros of $w$ are isolated in $G$, i.e. every zero has a neighbourhood $\Omega$ which does not contain other zeros of $w$, and in the neighbourhood $\Omega$ of a zero $y$ we have

$$
w(x)=\left[c(y)\left(x_{1}-y_{1}\right)+(-b(y)+i)\left(x_{2}-y_{2}\right)\right]^{m} w_{0}(x),
$$

where $m$ is a natural number and $w_{0} \in C^{0}(\Omega)$ does not vanish at $y$.

Proof: Let us denote by $W=U+i V$ the locally homeomorphic solution of the Beltrami equations. According to Theorem 1.2.2 we can assume that

$$
W(y)_{x_{1}} \neq 0 .
$$

By Lemma 1.3.1 we have in some neighbourhood $B(y, R)$ of the zero $y$ of $w$

$$
w(x)=(W(x)-W(y))^{m} \Psi(x),
$$

where $\Psi$ is twice continuously differentiable in $B$ and $\Psi(y) \neq 0$. The mapping $W$ has the Taylor expansion

$$
W(x)-W(y)=W(y)_{x_{1}} h+W(y)_{x_{2}} k+\frac{1}{2}\left(W_{11} h^{2}+2 W_{12} h k+W_{22} k^{2}\right),
$$

where $h=x_{1}-y_{1}, k=x_{2}-y_{2}, \quad$ and

$$
W_{i j}=U\left(y^{\prime}\right)_{x_{i} x_{j}}+i V\left(y^{\prime \prime}\right)_{x_{i} x_{j}}, \quad i, j=1,2 .
$$

The points $y^{\prime}$ and $y^{\prime \prime}$ are situated on the line segment joining $x$ and $y$. Because $W$ is a solution of (2.1.1) in $B$, we get

$$
\begin{gathered}
w(x)=[c h+(-b+i) k]^{m}\left[\frac{W(y)_{x_{1}}}{c}+\frac{W^{\prime}}{c h+(-b+i) k}\right]^{m} \Psi(x), \\
W^{\prime}=\frac{1}{2}\left(W_{11} h^{2}+2 W_{12} h k+W_{22} k^{2}\right) .
\end{gathered}
$$

But

$$
\left|W(y)_{x_{1}} / c(y)\right|>0
$$

and

$$
\left|\frac{W^{\prime}}{c h+(-b+i) k}\right| \leqq \gamma(|h|+|k|), \quad \gamma=\text { constant },
$$


A. I. 402

for the quadratic form

$$
|c h+(-b+i) k|^{2}
$$

is positive definite, since its determinant is $c^{2}>0$. Hence the second factor in (2.2.2) is different from zero in $B(y, R)$, when $R$ is small enough. This proves the lemma.

The natural number $m$ in (2.2.1) is called the order of the zero $y$.

If $f$ is a continuous mapping from a Jordan curve $C$ into $\mathbf{R}^{2}$, then we denote by

$$
\frac{1}{2 \pi} \Lambda_{C, x} \arg f
$$

the winding number of $f$ about the point $x \in \mathbf{R}^{2}-f(C)$ (cf. [11] p. 83).

Lemma 2.2.2. (The principle of argument.) Let $w=u+i v$ be pseudoanalytic on a Jordan region $G$. If $u$ and $v$ belong to $C^{0}(\bar{G})$ and $w \mid \partial G$ is not zero at any point, then $w$ has only a finite number $N$ of zeros in $G$ and

$$
N=\frac{1}{2 \pi} \Delta_{\partial G, 0} \arg w
$$

where all zeros are counted according to their orders.

Proof: Because $w \mid \partial G$ has no zero, Lemma 2.2.1 shows that $w$ can only have a finite number of zeros in $G$.

Let $x^{1}, x^{2}, \ldots, x^{N}$ be the zeros of $w$, every zero repeated according to its order. By Lemma 2.2.1 we have

$$
w(x)=w^{\prime}(x) \prod_{j=1}^{N}\left[c\left(x^{j}\right)\left(x_{1}-x_{1}^{j}\right)+\left(-b\left(x^{j}\right)+i\right)\left(x_{2}-x_{2}^{j}\right)\right] .
$$

where $w^{\prime} \in C^{0}(\bar{G})$ has no zero, hence

$$
\frac{1}{2 \pi} \Delta_{\partial G, 0} \arg w^{\prime}=0 .
$$

If we denote

$$
T_{j}(x)=c\left(x^{j}\right)\left(x_{1}-x_{1}^{j}\right)+\left(-b\left(x^{j}\right)+i\right)\left(x_{2}-x_{2}^{j}\right),
$$

then

$$
\frac{1}{2 \pi} \Delta_{\partial G, 0} \arg T_{j}=1
$$

since the mappings $T_{j}, j=1,2, \ldots, N$, are one-to-one, orientation preserving, continuous transformations of $\mathbf{R}^{2}$ onto itself, which take the points $x^{j}$ into origin. Thus (2.2.3) follows by the well-known properties of the winding number (cf. [11] p. 58). 
Corollary 2.2.1. If $w=u+i v$ is pseudoanalytic on a Jordan region $G$, $u$ and $v$ belong to $C^{0}(\bar{G})$, and $w \mid \partial G$ is a homeomorphism from $\partial G$ onto $\partial w(G)$, then $w$ is injective.

Proof: Let $w(y)$ be a point in $w(G)$. In view of Lemma 1.3.1 it is clear that $w(G)$ contains no point of $\partial w(G)$. Therefore

$$
\frac{1}{2 \pi} \Delta_{\partial G, w(y)} \arg w=1 .
$$

By Lemma 2.2.2 the mapping $w$ has in $G$ only one point where it takes the value $w(y)$, hence $w$ is one-to-one, and the corollary is proved.

Lemma 2.2.3. Suppose that $G$ is a Jordan region, $w=u+i v$ belongs to $B(G), \quad v \in C^{0}(\bar{G})$, and $u \mid \partial G=g \in C^{0}(\partial G)$. If the function $g$ has only one maximum and one minimum on $\partial G$ and is otherwise strictly decreasing or increasing, then

$$
w: \bar{G} \rightarrow \overline{w(G)}
$$

is a homeomorphism.

Proof: In view of Corollary 2.2.1 we must show that $w \mid \partial G$ is a homeomorphism.

According to the properties of $g, w$ is not constant. Therefore by Lemma 1.3.1, $w(G)$ is a region. By the same lemma the inverse image of $\partial w(G)$ belongs to $\partial G$.

Next we show that the image of $\partial G$ belongs to $\partial w(G)$. Suppose $x$ is a point on $\partial G$ such that

$$
w(x)=u(x)+i v(x)=g(x)+i v(x)
$$

belongs to $w(G)$. Because the inverse image of $\partial w(G)$ belongs to $\partial G$, there exist on $\partial G$ two distinct points $y$ and $z$ such that

$$
g(y)=g(z)=g(x)
$$

and $w(y)$ and $w(z)$ belong to $\partial w(G)$. But by the properties of $g$ either $y=x$ or $z=x$, hence $w(x)$ belongs to $\partial w(G)$.

Let $z^{1} \quad$ (resp. $z^{2}$ ) be the point where $w$ takes its minimum (resp. maximum). By assumption $\partial G$ consists of two simple closed Jordan ares $C_{1}$ and $C_{2}$, which have only the points $z^{1}$ and $z^{2}$ in common. It is clear that $w \mid C_{j}, j=1,2$, is an one-to-one continuous mapping from $C_{j}$ onto $w\left(C_{j}\right)$. Because $w(G)$ is connected, $w\left(C_{1}\right) \cap w\left(C_{2}\right)$ consists only of the points $w\left(z^{1}\right)$ and $w\left(z^{2}\right)$, hence $w \mid \partial G$ is a homeomorphism onto $\partial w(G)$. The lemma is proved.

Remark 2.2.1. Under certain conditions on $g$ and $G$ the function $v$ belongs to $C^{0}(\bar{G})$. For instance if $g$ is Hölder-continuous on $\partial G$ and there exists a twice Hölder-continuously differentiable mapping from $\partial B(0,1)$ into $\mathbf{R}^{2}$, which defines the boundary of $G$, then $v \in C^{0}(\bar{G})$ (cf. [3] p. 264). 


\subsection{General case}

In this section we suppose that $g$ satisfies the assumptions made in Lemma 2.2.3. Let $w=u+i v$ be pseudoanalytic on $G$ and $u \mid \partial G=g$. We want to show that under no conditions on $v$, the mapping $w$ is injective.

At first we study properties of the level lines of a pseudoharmonic function $u$. Let $J_{j}, j=1,2, \bar{J}_{1} \cup \bar{J}_{2}=\partial G$, denote the open Jordan arcs with the minimum point $z^{1}$ and the maximum point $z^{2}$ of $g$ as endpoints.

We need the following elementary lemma (cf. [8] p. 8) concerning the level lines of a harmonic function $h$.

Lemma 2.3.1. Let $h$ be harmonic on a region $F$ and not constant. Then for each point $x \in F$ there exists a neighbourhood $\overline{B(x, R)} \subset F$ and $a$ homeomorphism

$$
\Psi: \overline{B(x, R)} \rightarrow \overline{B(0,1)}
$$

such that $\Psi(x)=0$ and the image of the set

$$
h^{-1}(h(x)) \cap \overline{B(x, R)}
$$

under $\Psi$ consists of $2 m$ ( $m \geqq 1, m$ depending on $x$ ) rays from the origin.

Corollary 2.3.1. If $u$ is pseudoharmonic and not constant on $F$, then the above result holds.

Proof: Apply Lemma 2.3.1 to the function $h=: u \circ W^{-1}$, which is harmonic on the image of $W$.

Lemma 2.3.2. Let $u \in C^{0}(\bar{G})$ be pseudoharmonic on $G$ and $u: \partial G=g$. Then

a) $u^{-1}(r)$ is empty, if $r>g\left(z^{2}\right)$ or $r<g\left(z^{1}\right)$,

b) $u^{-1}\left(g\left(z^{j}\right)\right)=z^{j}, j=1,2$, and

c) $u^{-1}(r)$ is a closed Jordan arc in $\bar{G}$ with end points on $J_{1}$ and $J_{2}$, if $g\left(z^{1}\right)<r<g\left(z^{2}\right)$.

Proof: Assertions a) and b) are direct consequences from the maximum principle.

Let $x$ be a point in $G$. The set $u^{-1}(u(x))$ is a compact set in $\bar{G}$. We denote by $U_{x}$ the connected component of $u^{-1}(u(x))$ which contains the point $x$.

By Corollary 2.3.1 there exists $2 m(m \geqq 1)$ distinct points $x^{1}, x^{2}, \ldots, x^{2 m}$ on $\partial \Psi^{-1}(B(0,1 / 2))$ such that

$$
u^{-1}(u(x)) \cap \partial \Psi^{-1}(B(0,1 / 2))=\left\{x^{1}, x^{2}, \ldots, x^{2 m}\right\} .
$$


Let us denote by $U_{x}, j=1,2, \ldots, 2 m$, the connected component of

$$
u^{-1}(u(x)) \cap\left(\bar{G}-\Psi^{-1}(B(0,1 / 2))\right)
$$

which contains the point $x^{j}$. It is clear that $U_{x^{j}} \subset U_{x}$ and

$$
U_{x}=K \cup\left(\bigcup_{j=1}^{2 m} U_{x j}\right)
$$

where

$$
K=\Psi^{-1}(\overline{(B(0,1 / 2)}) \cap u^{-1}(u(x)) .
$$

By Corollary 2.3.1, the set $U_{x^{j}}$ contains points other than $x^{j}$.

We shall show that $U_{x^{j}} \cap U_{x^{k}}$ is empty, if $j \neq k$. Suppose that there exists a point $y$ in $U_{x^{j}} \cap U_{x^{k}}$. In this case at least one component $K^{\prime}$ of

$$
G-K \cup U_{x j} \cup U_{x k}
$$

contains a region, namely the inverse image under $\Psi$ of the set between rays leading from the origin to the points $\Psi\left(x^{j}\right)$ and $\Psi\left(x^{k}\right)$. But on $\partial K^{\prime} \subset u^{-1}(u(x))$ the function $u$ is equal to $u(x)$, and hence by the maximum principle $u$ is constant. This is a contradiction.

Next we show that $U_{x} \cap \partial G, j=1,2, \ldots, 2 m$, is not empty. For if it is, then there exists a region $V$ in $G$ such that $\bar{V} \subset G$ and

$$
u^{-1}(u(x)) \cap \bar{V}=U_{x^{j}}
$$

To find such a domain $V$ is possible because other components of $u^{-1}(u(x))$ are bounded away from $U_{x j}$ by Corollary 2.3.1. According to the maximum principle $U_{x j}$ contains no closed Jordan curve, hence $U_{x j}$ does not separate $V$ (cf. [11] p. 33). Therefore $V-U_{x}$ is connected, and so on $V$, either $u \leqq u(x)$ or $u \geqq u(x)$, with the equality at some point of $V$, for $U_{x^{j}}$ contains other points than $x^{j}$. But this is impossible by the maximum principle.

On $\partial G$ there exist two different points $y^{1} \in J_{1}$ and $y^{2} \in J_{2}$ with the property

$$
u\left(y^{k}\right)=g\left(y^{k}\right)=u(x), \quad k=1,2 .
$$

Because $U_{x^{j}} \cap U_{x^{k}}$ is empty, if $j \neq k$, and $U_{x^{j}} \cap \partial G$ is not empty, there exist exactly two sets $U_{x^{1}}$ and $U_{x^{2}}$, i.e. $m=1$.

The above argument also shows that $u^{-1}(u(x))=U_{x}$.

Every point $y \in U_{x} \cap G$ has an open neighbourhood $U$ in $U_{x}$, which is an open Jordan arc, hence every $y$ separates $y^{1}$ and $y^{2}$ in $U_{x}$. Because $U_{x}$ is a continuum, $U_{x}$ is a Jordan arc joining $y^{1}$ and $y^{2}$ (cf. [11] p. 27, Theorem (4.2)). The theorem is proved, for $u^{-1}(r)=U_{x}, r=u(x)$.

As a main result in this section we can now prove 
Theorem 2.3.1. If $w=u+i v \in B(G)$ and $u \mid \partial G=g$, then the mapping $w$ is injective.

Proof: By Lemma 2.3.2 there exists a homeomorphism

$$
\Phi: \bar{G} \rightarrow \overline{B(0,1)}
$$

such that

$$
\Phi\left(z^{1}\right)=-1, \Phi\left(z^{2}\right)=1
$$

and if $g\left(z^{1}\right)<r<g\left(z^{2}\right)$ then the set $\Phi\left(u^{-1}(r)\right)$ is the part contained in $\overline{B(0,1)}$ of the straight line perpendicular to the $x_{1}$-axis. Let now $x$ and $y$ belong to $G$ and $x \neq y$. Then there exists $\varepsilon>0$ such that $\Phi(x)$ and $\Phi(y)$ are contained in $B(0,1-\varepsilon)$. But on $\partial \Phi^{-1}(B(0,1-\varepsilon))$ the function $u$ has the same properties as $g$ has on $\partial G$. Moreover $v \mid \Phi^{-1}(\overline{B(0,1-\varepsilon)})$ is continuous. Applying Lemma 2.2 .3 to the mapping $w \mid \Phi^{-1}(\overline{B(0,1-\varepsilon)})$ we get $w(x) \neq w(y)$, which proves the theorem.

Remark 2.3.1. The above theorem can be used to guarentee the existence of quasiconformal mappings with prescribed once uniformly Hölder-continuously differentiable dilatation.

University of Helsinki

Helsinki, Finland 


\section{References}

[1] Ahlfors, L., and L. Bers: Riemann's mapping theorem for variable metrics. - Ann. of Math. 72 (1960), pp. 385-404.

[2] Акô, K.: On Perron's process associated with second order elliptic differential equations. - J. Fac. Sci. Univ. Tokyo Sect. I. 9 (1960), pp. 165-202.

[3] Bers, L., and M. Schechter: Elliptic equations. - Partial differential equations, Lectures in Applied Mathematics 3, Interscience Publishers, New York/ London/Sydney, 1964, pp. 131-300.

[4] Hellwig, G.: Partielle Differentialgleichungen. - Mathematische Leitfäden, B. G. Teubner Verlagsgesellschaft, Stuttgart, 1960.

[5] Lehto, O., und K. I. Virtanen: Quasikonforme Abbildungen. - Die Grundlehren der mathematischen Wissenschaften 126, Springer-Verlag, Berlin/ Heidelberg/New York, 1965.

[6] Miranda, C.: Equazioni alle derivate parziali di tipo ellittico. - Ergebnisse der Mathematik und ihrer Grenzgebiete, Neue Folge 2, Springer-Verlag, Berlin/Göttingen/Heidelberg, 1955.

[7] Monrey, C. B.: On the solutions of quasilinear elliptic partial differential equations. - Trans. Amer. Math. Soc. 43 (1938), pp. 126-166.

[8] Monse, M.: Topological methods in the theory of functions of a complex variable. - Princeton University Press, Princeton, 1947.

[9] Perron, O.: Eine neue Behandlung der ersten Randwertaufgaben für $\Delta u=0$. - Math. Z. 18 (1923), pp. $42-54$.

[10] Vekua, N.: Generalized analytic functions. - Pure and Applied Mathematics 25, Pergamon Press, Oxford/London/New York/Paris, 1962.

[11] Whyburn, G.: Topological analysis. - Princeton Mathematical Series 23, Princeton University Press, Princeton, 1964. 